\title{
Observations on student difficulties with mathematics in upper-division electricity and magnetism
}

\author{
Rachel E. Pepper, Stephanie V. Chasteen, Steven J. Pollock, and Katherine K. Perkins \\ Science Education Initiative and Department of Physics, University of Colorado, Boulder, Colorado 80309, USA
}

(Received 28 July 2011; published 27 March 2012)

\begin{abstract}
We discuss common difficulties in upper-division electricity and magnetism (E\&M) in the areas of Gauss's law, vector calculus, and electric potential using both quantitative and qualitative evidence. We also show that many of these topical difficulties may be tied to student difficulties with mathematics. At the junior level, some students struggle to combine mathematical calculations and physics ideas, to account for the underlying spatial situation when doing a mathematical calculation, and to access appropriate mathematical tools. We discuss the implications of our findings for E\&M instruction at the junior level.
\end{abstract}

DOI: 10.1103/PhysRevSTPER.8.010111

PACS numbers: 01.40.Fk, 01.30.lb, 01.40.gb

\section{INTRODUCTION}

There is a wealth of detailed knowledge about specific student difficulties in physics at the introductory level (see McDermott and Redish [1] for a good summary). However, research into student difficulties at the upper division is just beginning [2-11]. At the University of Colorado (CU) we are engaged in a multiyear project to transform some of our upper-division courses through action research [12-14], including identification of student difficulties, creation of learning goals for the courses, and integration of studentcentered instruction $[9,10,15-22]$. As part of this broader goal, we have been documenting student difficulties in the first semester of our junior-level electricity and magnetism $(\mathrm{E} \& \mathrm{M})$ course sequence for the past six semesters [9].

In upper-division physics courses, including E\&M, it becomes increasingly important for students to use sophisticated mathematical calculations and concepts and integrate them with physical understanding. We have observed that achieving this math-physics integration consistently is a difficult and slow process for many students. Thus, we aim to document an initial list of common student difficulties with the goal of providing resources for improvement of instruction and for future research. When identifying such difficulties we define mathematics in a broad sense as encompassing not just calculations (algebra, performing integrals, taking derivatives) but also including thinking about geometry, symmetry, vector calculus, and integrals (both vector and scalar) and the interpretation of calculations.

In the present investigation, we focus on student reasoning and ideas closer to the scale of concepts rather than the more fine-grained ideas of resources [23-25]. Concepts are

Published by the American Physical Society under the terms of the Creative Commons Attribution 3.0 License. Further distribution of this work must maintain attribution to the author(s) and the published article's title, journal citation, and DOI. typically described as stable well-articulated ideas students have about how the world works, such as that current gets used up in a circuit. Resources, on the other hand, are more general and finer grained, such as "more cause leads to more effect." Resources can be put together in different combinations by a student depending on context; for instance, more push means more motion. In this paper our focus is most closely aligned with "concepts," since we believe that by looking at the ways students talk about and employ mathematical ideas in the context of our upperdivision E\&M course, we may identify some persistent behaviors and common difficulties which will prove valuable in ongoing development of instructional strategies and curricular materials. We seek to identify student responses and the corresponding student difficulties that appear across common classroom, homework, and exam problem-solving contexts, and which are stable across time. Rather than attempting to provide an exhaustive survey of conceptual difficulties in junior E\&M, this work focuses on the mathematical difficulties that were most prevalent across students and contexts within our observations.

Other research studies have investigated student difficulties with mathematics in other upper-division courses. In an upper-level thermodynamics context, Meltzer has observed that students have difficulty connecting $P-V$ diagrams of different processes to the work done by the system during the process [5]. Pollock et al. further showed that most students who answer these $P-V$ diagram questions incorrectly are having trouble connecting an integral to the spatial idea of the area under a curve [4]. In an upperlevel thermodynamics course, Loverude discusses student difficulties with probability concepts, which are related to student difficulties accessing an appropriate tool for solving the problem [6]. In an intermediate mechanics course, Hayes and Wittmann observed students having difficulty combining physics ideas with mathematical calculations when choosing plus and minus signs to set up $F=m a$ calculations [8]. There is also evidence that students have 
trouble interpreting equations in an upper-division chemistry course [26].

Bing and Redish suggest that some of these mathematical difficulties may arise from issues of epistemological framing, which cause students to selectively attend to particular elements of their math knowledge while ignoring other (potentially more useful) elements [27]. Dray and Manogue point out that some student struggles could be tied to a gap between how mathematicians, who teach the math prerequisites for physics classes, and physicists think about math [28,29]. Redish also observes that even in physics classes we often focus students' attention on the process of solving mathematics and do not emphasize model making and interpreting results [30].

With this prior work in mind, we focus here on an investigation of underlying mathematical issues demonstrated by students in a variety of contexts within six semesters of one upper-division electromagnetism course. In this paper we address three research questions, motivated by our interest in providing resources for improvement of instruction, and as a base for future research: (1) Do we find evidence of the same kinds of challenges in mathematics and math-physics connections as has been observed in lower-division (e.g., McDermott and Redish [1], Sec. IV.D, and others [31-34]) and other upper-division physics contexts? (2) What are some common, recurring ideas and difficulties rooted in mathematics underpinning the canonical topical areas of a typical first upper-division electromagnetism course? (3) Given this preliminary catalog of student difficulties, can we categorize these difficulties?

In these studies, we concentrate on three topical areas: Gauss's law, vector calculus, and electric potential. These areas span a significant portion of a canonical upperdivision E\&M course and form a rich starting point for observing student use of, and issues with, math and mathphysics connections in this junior-level course. We present evidence in each of these areas that there are indeed common math-physics difficulties which can be identified and categorized. These data then suggest several categories of common difficulties with mathematics from students at the junior level:

- Students have difficulty combining mathematical calculations and physics ideas. This can be seen in student difficulty setting up an appropriate calculation and also in interpreting the results of the calculation in the context of a physics problem. (However, students can generally perform the required calculation.)

- Students do not account for the underlying spatial situation when doing a mathematical calculation.

- Students do not access an appropriate mathematical tool. Students may instead choose a mathematical tool that will not solve the relevant problem, or may choose a tool that makes the problem too complex for the student to solve.

\section{COURSE DESCRIPTION AND METHODS}

For this study we documented student difficulties in the first semester of a two-semester E\&M sequence at CU. This course is offered every semester, uses Griffiths' E\&M textbook [35], and typically enrolls between 35 and 60 students. The material covered in the first semester includes electrostatics and magnetostatics (the first six chapters of Griffiths' textbook). We have recently transformed this course so that it includes many techniques based on physics education research, such as peer instruction [36], and weekly tutorials [37]. Of the six semesters we have been collecting data on this course at $\mathrm{CU}$, five semesters are of the transformed implementation [15].

For the six total semesters at $\mathrm{CU}$, the course has been composed of, on average [38], $47 \% \pm 5 \%$ physics majors, $31 \% \pm 4 \%$ engineering physics majors, and has been $22 \% \pm 3 \%$ female. Students typically come into this course with an average overall grade-point average of $3.12 \pm 0.02$. The small standard errors show that the demographics of this course are consistent across all six semesters. There is, however, a small difference in demographics between the fall and spring semesters of this course: spring semesters tend to be larger and to be composed of more physics majors and fewer engineering physics majors.

We employed a mixed methods approach in our research, combining quantitative and qualitative [39] data as described below. Mixed methodology is appropriate because we seek student ideas that arise in multiple content areas and across multiple data sources. It is also practical, since we are performing action research integrated with course transformation, and have access to student work, the chance to observe students in course-related settings, but limited resources to perform in-depth ethnographic studies of students. We used emergent coding schemes [39] to analyze student interviews as well as student written explanations on exams and diagnostics. The specific student difficulties that we focus on in this paper emerged from a synthesis of the full set of data and observations. We chose those difficulties that were prevalent, central to the course learning goals, related to mathematics, and which had sufficient evidence to warrant deeper analysis.

For each semester, we administered and analyzed results from the Colorado Upper-Division Electrostatics (CUE) diagnostic, a conceptual survey of electrostatics (and some magnetostatics) designed to test learning goals that our faculty value [17]. The questions are open ended and graded with a detailed rubric for both correctness and explanation. We also have CUE results for several E\&M courses at other institutions. We have, additionally, collected midterm and final exam solutions and weekly conceptually focused tutorial pre- and posttests. Midterm and final exam scores listed in this paper come from grading by a single grader using a detailed rubric created by us.

We have also taken extensive field notes during interactive lectures, homework help sessions, and tutorials. 
Both the homework help sessions and the weekly tutorials are focused on students working in small groups, so are ideal to observe student conversations and externalization of student thinking.

Additionally, we conducted several sets of video-taped, individual student interviews where students were asked to solve E\&M problems in a think-aloud protocol [40]. Interviews used a semistructured protocol which included follow-up and clarification questions. Interviews include three interviews in which students enrolled in a transformed E\&M 1 course were asked a series of questions about Ampère's law and Gauss's law, four interviews in which students who had completed a transformed E\&M 1 course in the previous semester were asked a series of questions about Gauss's law followed by one question about electric potential, and 11 interviews in which students who were either currently enrolled in a transformed E\&M 1 course or had taken one recently took the CUE diagnostic while thinking aloud with no interviewer intervention. Think-aloud protocols were chosen because they have the advantage of providing an opportunity to observe student behavior similar to that in a relevant context (e.g., doing homework) and are able to target student responses to an area of interest to us as researchers.

\section{GAUSS'S LAW}

Gauss's law, $\oint \mathbf{E} \cdot \mathbf{d A}=Q_{\text {enc }} / \epsilon_{0}$, is commonly taught in both introductory and upper-level physics courses. It has been shown through student interviews and a diagnostic exam that, in introductory physics courses, students struggle with Gauss's law [41]. Anecdotally, we find most instructors do not expect that juniors taking an $\mathrm{ad}$ vanced course in electricity and magnetism will also have significant difficulties with Gauss's law. However, Singh uses the same diagnostic exam to show that traditionally taught upper-level students do have difficulties with Gauss's law similar to the introductory-level difficulties [41]. Here, we follow up on these results and probe the upper-division student difficulties further using interviews. Using evidence from the CUE diagnostic [17], exam questions, and student interviews, we observe that in both transformed and traditionally taught courses at CU even the best juniors still struggle with aspects of Gauss's law and that many of these struggles appear to be connected to the difficulties with mathematics mentioned earlier.

For reference when considering student difficulties, we review the correct logic for using Gauss's law. For Gauss's law to be directly useful one must (1) be able to determine from the symmetry of the charge distribution what direction $\mathbf{E}$ points and on what variables $\mathbf{E}$ depends so that one can (2) create a Gaussian surface on which $\mathbf{E} \cdot \mathbf{d} \mathbf{A}$ is known to be either constant or zero. Once such a Gaussian surface has been created, one can then (3) solve for $\mathbf{E}$ by pulling it out of the integral.

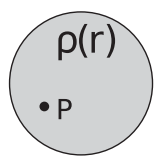

(a)

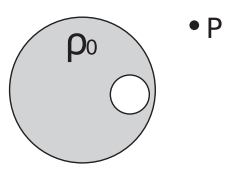

(b)
FIG. 1. Illustrations from CUE diagnostic questions. Students are asked not to solve the question, but to give "the easiest method you would use to solve the problem" (half credit) and "why you chose that method" (half credit). (a) "A solid nonconducting sphere, centered on the origin, with a non-uniform charge density that depends on the distance from the origin, $\rho(r)=\rho_{0} e^{-r^{2} / a^{2}}$. Find $E$ (or $V$ ) at point $P$." (b) "A charged insulating solid sphere of radius $R$ with a uniform volume charge $\rho_{0}$, with an off-center spherical cavity carved out of it. Find $E$ (or $V)$ at point $P$, at a distance $4 R$ from the sphere."

\section{A. Quantitative evidence that students struggle}

Singh presents evidence that both introductory and upper-level students do poorly on a Gauss's law diagnostic which tests understanding of concepts important for applying Gauss's law: both score $49 \%$ postinstruction $\left(N_{\text {lower }}=541, N_{\text {upper }}=28\right)$. In contrast, graduate students, who can be considered more expert, exhibit better mastery, scoring $75 \%(N=33)$ [41].

We also see evidence of some students struggling with Gauss's law after junior-level E\&M 1 in the results of the CUE diagnostic. Of the 324 total students who took the CUE after an E\&M 1 course, 33\% did not recognize a radially symmetric problem as most easily solved with Gauss's law [see Fig. 1(a) for the full problem]. Of the $67 \%$ who correctly identified Gauss's law as the easiest solution technique, the average score for explanation of why and how Gauss's law was used was 59\%. The explanation score included points for specifying the correct Gaussian surface as well as for explaining why using Gauss's law was appropriate, for instance, discussing what symmetries are present in the problem.

In a second CUE question, involving a problem without sufficient symmetry for Gauss's law to be directly useful, students make the opposite mistake, and misidentify direct application of Gauss's law as an appropriate technique [see Fig. 1(b) for the full problem]. Of 324 students, 24\% incorrectly chose Gauss's law as the easiest technique for this problem, despite the fact that a Gaussian surface where $\mathbf{E} \cdot \mathbf{d A}$ is constant or zero cannot be created. Overall, $46 \%$ of the 324 students who took the exam made at least one of these two mistakes related to Gauss's law.

Students also revealed difficulties with Gauss's law on a midterm exam question asked in three different semesters of E\&M 1 at CU. This question was as follows:

Suppose I evenly fill a cube (length $L$ on a side) with electric charges. Then imagine a larger, closed cubical surface neatly surrounding this cube (length $2 L$ on a side). A) Is Gauss's law TRUE in this situation? (Briefly, why or why not?) B) Can one use Gauss's law to simply compute the value of the $E$ field at 
arbitrary points outside this charged cube? (Don't try, just tell me if you could, and why or why not?)

In a recent semester of a transformed course, most students correctly answered "yes" to the first part of the question, with an average score for that part of $89 \%$ including points for both answer and explanation $(N=59)$. However, on the second part of the question students scored, on average, only $46 \%$, including points for both answer and explanation. Nearly a third of students received no points at all for their answer to part (B) of this question. Some common student responses will be discussed below in Sec. III B 1.

\section{B. Insight from interviews}

In order to understand why upper-level students struggle with Gauss's law we conducted think-aloud interviews in which four students who had completed an E\&M 1 course in the previous semester were asked a series of questions about Gauss's law.

Below we discuss several difficulties observed during the course of these interviews and connect them to possible student difficulties with mathematics. Because of the small sample size of students interviewed (who all got A's and B's in $\mathrm{E} \& \mathrm{M} 1$ ), we do not attempt to generalize to the larger population of upper-division E\&M students. However, this small-scale qualitative approach is complementary to the larger-scale quantitative results provided by course exams and the CUE and allows us to describe in detail some of the possible difficulties of upper-division students.

\section{Incorrect inferences about $\mathrm{E}$ based on the flux and an inverse problem}

Gauss's law is more subtle mathematically than many equations that students have encountered up to this point in their education in the sense that it is an inverse problem. Students are accustomed to problems in which it is possible to use algebra to get the quantity of interest by itself on one side of the equals sign. For Gauss's law, the quantity of interest, the electric field, is embedded inside an integral and several steps of reasoning are necessary before the integral can (sometimes) be replaced with an algebraic expression. The similarly difficult nature of Ampère's law as an inverse problem is discussed by Manogue et al. [42]. The inverse nature of Gauss's law seems to cause some student difficulties.

Two of the four students interviewed had the same difficulty when addressing the problem of an unevenly shaped insulator of uniform charge density, $\rho$. These students incorrectly inferred from Gauss's law that the electric field at any point on a Gaussian surface inside the insulator was determined only by the charge enclosed, ignoring the distribution of the charge. Both students also did not clearly distinguish between the electric field at a single point on the surface and the flux through the entire surface in their explanations. This failure to distinguish the two in words may indicate that these students also do not clearly differentiate the ideas of flux and electric field, which may be partially leading to their struggle to correctly apply Gauss's law.

The first student claimed that

... the only thing that determines the flux out of it [the Gaussian surface] is the total enclosed charge. And so, if we took a sphere, and filled it completely with a completely uniform charge density in a spherical shape [draws an isolated solid circle with arrow's pointing outwards and spaced uniformlysee (B) on the left side of Fig. 2], say, then we could get a flux coming out of that area ... 'cause then it would be uniform.

When asked if the electric field would be the same for the spherical Gaussian surface drawn inside the blob of insulator as for the isolated sphere she had drawn during her explanation, this student claimed "Yes. Because Gauss's law shows us that only the enclosed charge, um, matters." She proceeded to explain that this was the case because " $E$ field lines only start and end on charges" so the lines from a point charge external to the Gaussian surface will pass through "so the only contributions that matter are the enclosed ... charge contributions." It seems that she has inappropriately inferred that, because the flux depends only on the enclosed charge, the electric field will be uniform and radial on any spherical surface inside any insulator with uniform charge density $\rho$. Here she is also not specific about whether these contributions are to the flux through the Gaussian surface or to the electric field at an arbitrary point on the Gaussian surface.

Another student brought up the situation of an uneven shape of constant $\rho$ on his own when asked to give examples of situations where Gauss's law is and is not useful for finding $\mathbf{E}$. He used the uneven shape as an example where you could use Gauss's law to find the electric field.

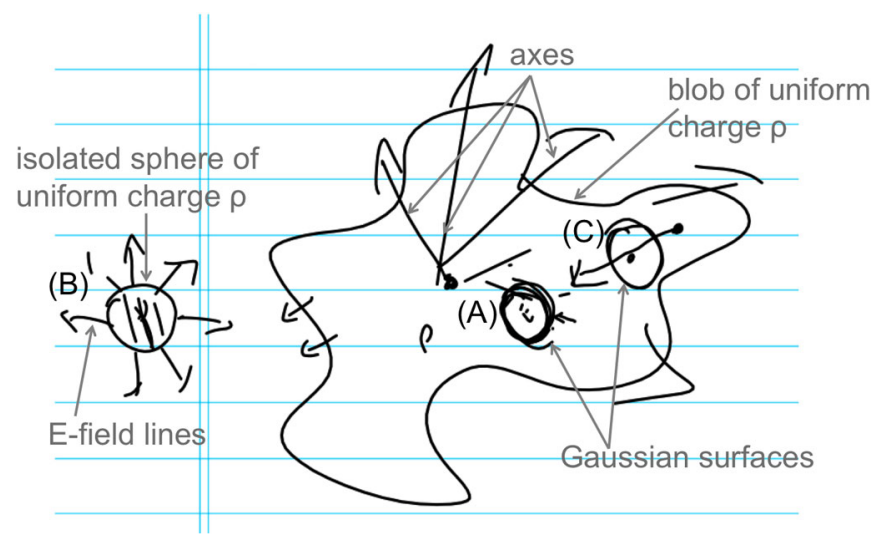

FIG. 2 (color online). Student drawing from Gauss's law interview. After drawing axes and the nonuniform blob of charge, the student drew the left-hand Gaussian surface, (A), and explained as detailed in the text. The student next drew the isolated sphere of charge, (B), to help explain her point, followed by the righthand Gaussian surface, (C). 
He explained that "the $E$ field ... that passes through a Gaussian surface is only dependent on the $Q$ enclosed." He then uses this statement to justify that one can find $\mathbf{E}$ using Gauss's law for a Gaussian surface inside the shape:

On the inside, once again if it's $[\rho$ is] constant, then that's fine, because there's ... because it doesn't matter what the shape is looking like 'cause we're not looking on the outside. We're only looking ... it's only dependent on the $Q$ enclosed.

Here the student is not clear on what the "it" is that is only dependent on the $Q$ enclosed-he could be thinking (correctly) about the flux, or (incorrectly) about the electric field. This student later states that he thinks that $\mathbf{E}$ is the same throughout a Gaussian surface inside this object, but expresses some discomfort with his understanding, saying "'Cause if there's $Q$ on the outside, the charge, you know, is making an $E$ field as well ... and therefore it must affect the $\mathbf{E}$ field at that point [points to a point on the Gaussian surface] as well. So I'm still ... I'm still not really happy with Gauss's law."

That these students do not clearly articulate (or seem to acknowledge) the difference between flux through a surface and electric field at a point on the surface may indicate that these students are not accounting for the underlying spatial situation when using the mathematical formula for the flux. Were these students thinking about the flux as the sum of dot products of area vectors with the electric field across some geometrical surface, it is unlikely that they would confuse $\mathbf{E}$ and a surface integral involving E. A similar student difficulty has been seen in the context of Ampère's law, where students do not interpret a line integral as a sum [9].

The incorrect inferences that these students make about the electric field from Gauss's law's statement about flux through a surface further indicate that they are not connecting the idea of the integral to a physical situation. The difficulties are also similar to difficulties discussed by Singh [41], and by Wallace and Chasteen in regards to Ampère's law [9]. In both cases, the authors describe students incorrectly inferring that because the integral is zero, the field in the integrand is zero as well. The students we observe may also be using reasoning in which students consider the right-hand side of an equation the cause and the left hand side its effect [43] —in this case thinking that $Q_{\text {enc }}$ is the only cause of the electric field on the Gaussian surface, without thinking about the fact that the left-hand side of the equation is an equation for flux rather than electric field alone.

Our observations of these students discussing Gauss's law show that even students who earn A and B grades in the course may exhibit these difficulties after an E\&M course. It is notable that the two students discussed above were top students in their E\&M course; both received above 95\% for their overall course score. The problem of confusing the electric field and flux, or making incorrect inferences in the context of an inverse problem where $\mathbf{E}$ cannot be solved for algebraically, persists even among the best upper-level students.

We also occasionally observe a more basic problem with the inverse nature of Gauss's law. As is common for introductory students [41,44], a few upper-level students use Gauss's law in a rote way by just solving $E A=$ $Q_{\text {enc }} / \epsilon_{0}$ without considering symmetry or visualizing the electric field. This type of solution was seen in one interview (out of four that were focused on Gauss's law), and in work for solutions to the exam problem involving a cube, described in Sec. III A. In 13 out of 59 exams students say Gauss's law can be used to find $E$ simply, though in none do any students write specifically $E A=Q_{\text {enc }} / \epsilon_{0}$; when this exact question was repeated on a second midterm (given to the same students), 5 out of 51 students said Gauss's law can be simply used, and two of these clearly use logic equivalent to $E A=Q_{\text {enc }} / \epsilon_{0}$.

These students are not using an appropriate tool to think about this integral. In a purely mathematical context, it is likely that these students are aware of how to treat functions inside integrals since they have successfully completed a course in multivariable calculus. However, for some reason, in the context of Gauss's law, they do not apply this knowledge. It may be that students are applying an algorithm they have used successfully in the past, rather than first visualizing the problem physically or geometrically. This would be consistent with the idea that the difficulty these students are experiencing is one of epistemological framing [27] where these students are framing the problem as a calculation, when to correctly solve it, they must first frame it as a physical mapping. A similar difficulty has been seen for students calculating the magnetic field using Ampère's law: some students try to solve problems without first visualizing the magnetic field or drawing an Ampèrian loop [9].

\section{Difficulty with symmetry arguments}

In order to create a proper Gaussian surface, one must use the symmetry of the problem to determine what direction the electric field points and on which variables it depends. Many of the students interviewed could make these predictions in highly symmetric situations, but could not justify one or both of the direction or the dependence of the electric field.

In general there are two categories of symmetry arguments that experts use to justify the direction and the dependence of the electric field: geometrical symmetry arguments and superposition symmetry arguments. In geometrical symmetry arguments, the logic relies only on the geometry of the charge distribution. One student in the set of interviews about Ampère's law and Gauss's law makes such an argument when describing the electric field around a long charged cylinder with $\rho=K s$, where $s$ is the radial 
cylindrical coordinate: "If you rotate the cylinder, the $E$ field should be the same. So there can't be any $\phi$ dependence," and "if you go up and down it looks the same" so there cannot be any $z$ dependence. This argument can be extended to include the direction of the electric field by arguing that an infinite cylinder looks the same looking in the $+\hat{z}$ direction as in the $-\hat{z}$ direction, so that an electric field pointing in the $\hat{z}$ direction would be contradictory. An analogous argument can be made to eliminate a $\hat{\phi}$ component.

The second type of symmetry argument, based on superposition and Coulomb's law, can be employed to deduce the direction of the electric field. For instance, when considering the electric field at an arbitrary point above an infinite line charge, one can imagine that, for every small piece of the line to the right of the point of interest, there is another small piece of the line at the same distance from the point on the left, and that when the electric field from these two pieces are added at the point of interest, the horizontal components cancel, leaving only a radial component. In Griffiths' E\&M textbook [35], this type of superposition symmetry argument is fairly common; it is made directly in a worked example for an infinite line charge, and several problems are included where this technique greatly simplifies the solution-for instance, the electric field on the symmetry axis of rings, squares, and disks of charge ([35], pp. 62-64). On the other hand, Griffiths models a geometry-based symmetry argument only once ([35], p. 70) in the context of Gauss's law (and this in a footnote) and the other Gauss's law examples simply state the direction with statements like "by symmetry" or "symmetry dictates" ([35], p. 73).

Perhaps it should not be surprising, then, that the students we observe almost exclusively make superposition symmetry arguments, even when these arguments are unproductive. All four students who were asked about an infinite line of charge discussed only the lack of a $z$ component and made superposition symmetry arguments. When asked directly about why there was no $z$ dependence of the electric field in this situation, three of the students used a superposition symmetry argument that horizontal components of $\mathbf{E}$ cancel and leave only radial components, despite the fact that they had been asked about dependence, not direction. One of these students also tried to use a similar superposition argument to explain why the electric field points radially outward from a sphere. While this argument is possible, the student did not succeed, and from an expert's perspective, it may be easier to make this argument based on geometry rather than superposition of electric fields. Superposition symmetry arguments are the predominant type of symmetry arguments students used in our interviews.

Students also used superposition arguments when not applicable. For instance, one student discussed the use of Gauss's law near an unevenly shaped insulator with uniform $\rho$. He drew a Gaussian surface close to the surface of the object with the same shape as the object, and was trying to decide whether it was possible to use Gauss's law on that surface. He explains that he is trying to think what the electric field looks like by mentally adding up the contributions from the different parts of the shape. This is a difficult task, and the student ended up incorrectly deciding that the electric field was perpendicular to the Gaussian surface and uniform on the Gaussian surface. It is possible that, as in the case just described, students' misuse of superposition arguments when geometrical symmetry arguments are appropriate is leading to student difficulties figuring out when to apply Gauss's law in novel situations.

Intriguingly, none of the students interviewed made a complete argument for both the dependence and the direction of the electric field-perhaps because completely determining both is difficult without employing some geometry-based symmetry arguments.

Manogue et al. point out in the context of Ampère's law that even immediately after explicit instruction in expertlike geometrical symmetry arguments, students struggle to recreate them when solving a new problem [42]. This may indicate that these geometrical symmetry arguments are difficult and nonintuitive even for upper-division students.

The reliance of these students on superposition arguments to the near exclusion of geometry arguments when thinking about symmetry is an example of students not using appropriate mathematical tools and not being able to set up a problem given a physical situation. To properly set up and justify the approach to many Gauss's law problems, using both types of symmetry arguments is essential; therefore, neglecting one type of symmetry argument precludes using the appropriate tools for the solution.

\section{VECTOR CALCULUS}

In junior-level E\&M using vector calculus to understand and work with the electric and magnetic fields is critical. Students must regularly use vector derivatives and vector integrals, including line, surface, and volume integrals. Perhaps, even more importantly, one of our main goals for this course is for students to understand these operations as physical expressions about vector fields in space, rather than as abstract mathematical calculations. We discuss below student difficulties we have observed with vector calculus. We first discuss students struggling with the basic idea of a vector field, then discuss student difficulties with vector derivatives, and finally address student difficulties with vector integration. Many of these difficulties are examples of students not accounting for the underlying spatial situation when performing calculations and not using appropriate mathematical tools.

\section{A. Vector fields}

We observe that several students at the upper division have trouble with the vector nature of a vector field. We find that some students do not simultaneously attend to 
both the magnitude and direction of the vector, but rather concentrate on one aspect at a time, sometimes switching which aspect they attend to midproblem, or midinterview. We see these difficulties, in particular, when students are predicting the results of a sum of several vectors, or the result of a line, surface, or volume integral in a vector field. For instance, a student who received an A in E\&M 1, and was interviewed in one 60 min block, attended only to the magnitude of the electric field when answering one interview question, and attended to only the direction in a later question. When answering the midterm question about a cube described in Sec. III A, he thought only about the magnitude of the electric field. In the context of this question he explained that "In order for us to use this [Gauss's law], we need to find a surface that $\mathbf{E}$ is constant over this entire surface." He neglected to explain that the direction of $\mathbf{E}$ compared to $\mathbf{d A}$ also needed to be the same everywhere on the surface, even when the interviewer probed further by asking if there were any other criteria for the usefulness of Gauss's law. On the other hand, when asked a superposition question from Singh's Gauss's law diagnostic [41] later in the interview, he thought only about the direction of the $E$ field. The question asked was as follows:

Three identical point charges $+\mathrm{Q}$ are arranged in a line as shown above. Points A, B, and $\mathrm{C}$ are along a parallel line. You do not know the lengths $\mathrm{L}$ and $\mathrm{d}$. The three charges produce an electric field. Without knowledge of $\mathrm{L}$ and $\mathrm{d}$, what can you infer about the electric field at points A, B, and C?[41]

In answering this question, the student only discussed (and drew, as shown in Fig. 3) which direction the electric field would point and neglected the fact that the electric field would have different magnitude depending on the distance from each charge. Later in this same question he began thinking again about magnitude, when adding the vectors from the three charges to get a total electric field (far right of the figure).

This difficulty in simultaneously attending to both the direction and magnitude of a vector field may again be an example of students not connecting the math to the physical geometry-for these students, the concept of a vector field as a vector with direction and magnitude at every point in space is still somewhat tenuous and these students struggle to consistently apply this concept.

\section{B. Vector derivatives}

In junior-level electricity and magnetism, students must connect the vector derivatives (gradient, divergence, and curl) to their physical meaning in order to better understand Maxwell's equations. All students entering junior-level E\&M 1 have some previous experience with vector derivatives from Calculus 3, a required prerequisite taken in the math or applied math departments. Students entering E\&M 1 can successfully calculate these derivatives in Cartesian coordinates: When asked to compute the

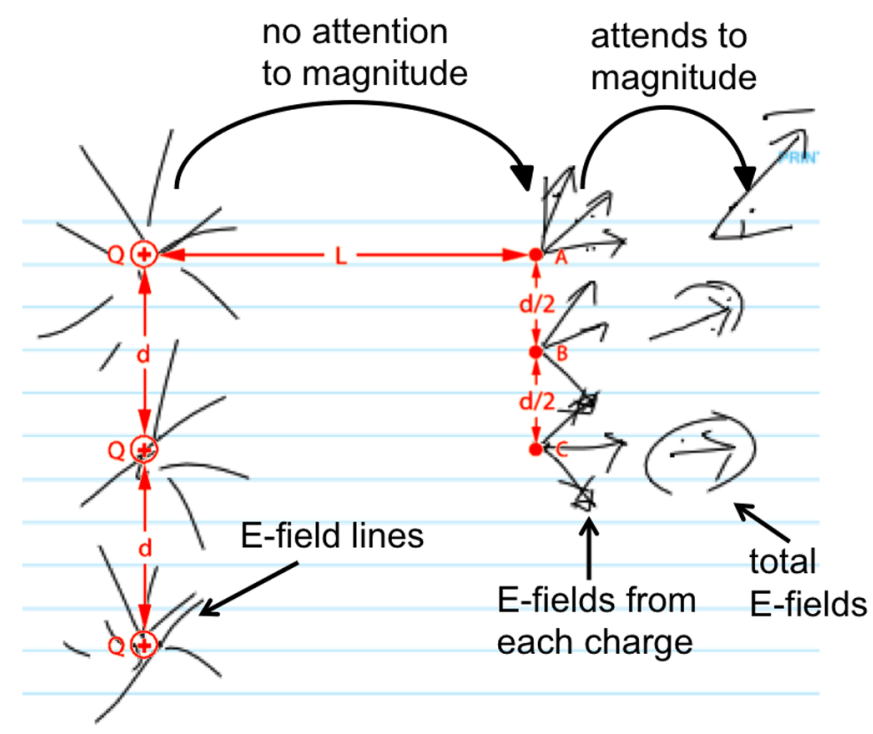

FIG. 3 (color online). Student drawing from interview. Portions of the picture in red are from the question statement which is reprinted with permission from Singh [41]. Dark gray is the student's drawing, and explanation of the student's drawing are black. After drawing electric field lines radiating outwards from each charge, the student drew three vectors of equal magnitude at each measurement point-one for the contribution to the electric field from each charge. At this point he does not attend to the magnitude of the vectors. To the right of that the student then drew the sum of those vectors as the total electric field. In this stage he attends to the magnitude of the vectors.

gradient of $e^{x} \cos (y)$ and $\cos \left(x^{2}+y^{2}+z^{2}\right)$ as a pretest in the first homework of the semester, students in two different semesters of E\&M $1(N=90)$ scored on average $90 \pm$ $3 \%$. When asked to compute the divergence and curl of $\hat{i}\left(x^{2}+y z\right)+\hat{j}\left(y^{2}+z x\right)+\hat{k}\left(z^{2}+x y\right)$ on the same homework, the same students scored on average $90 \% \pm 2 \%$. While students demonstrate calculational success, they come into the course with less understanding of the physical meaning of these calculations: on the same pretest homework the same students averaged $77 \% \pm 3 \%$ when asked to identify which out of four drawn vector fields "have nonzero divergence somewhere" and which "have nonzero curl somewhere."

The interpretation of these vector operations provides an example of students not accounting for the underlying spatial situation when performing calculations, and not using appropriate mathematical tools. We will focus in this section mainly on student thinking about divergence, but have made similar observations about student thinking about the gradient and curl vector operations.

The divergence has to do with the "sourceness" or "sinkness" of the vector field: it tells you if there is a source or sink at the point where the divergence is calculated. In electrostatics, sources and sinks come from electric charges, and the divergence of the electric field is 
therefore related to the charge density, $\rho: \nabla \cdot \mathbf{E}=\frac{\rho}{\epsilon_{0}}$. A more visual way to assess whether a particular point on an electric field line drawing has nonzero divergence is to draw a small closed surface in the field and determine if all the electric field lines that enter the circle also leave the circle. If this is true, the area inside is divergence free, if not, there is divergence at some point inside the circle.

While an expert connects the math of divergence to the spatial situation and to the physics of electric fields using either the visual method described above or $\nabla \cdot \mathbf{E}=\frac{\rho}{\epsilon_{0}}$, we find that students struggle with this idea in the context of electrostatics. For instance, 51 students in a transformed $\mathrm{E} \& \mathrm{M} 1$ class at $\mathrm{CU}$ were asked the following question on a midterm exam:

You have a thin, non-conductive spherical shell with radius $R$ centered at the origin. The shell carries a total charge $-Q$, which is uniformly distributed over the surface of the shell. There are no other charges anywhere. Where in space (if anywhere) does the divergence of $\mathbf{E}$ vanish?

Only $18 \%$ of students gave a full correct and explicit answer such as "The divergence of $\mathbf{E}$ is proportional to the charge density at any given point in space. In this system there is no charge anywhere except on the boundary of the sphere so the divergence of $\mathbf{E}$ vanishes everywhere except at $r=R$." Eight percent of students gave the correct, but less specific answer of "where there are no charges." The other $74 \%$ of the students did not get this question correct, and their answers are broken down in Fig. 4. The most common incorrect answer, given by $31 \%$ of the students, was that the divergence was zero only inside the sphere. While this is partially correct, these students did not realize that the divergence is also zero outside the sphere. We see similar results for the same question asked on a tutorial posttest in a different semester of this course $(N=18)$ and in our field observations.

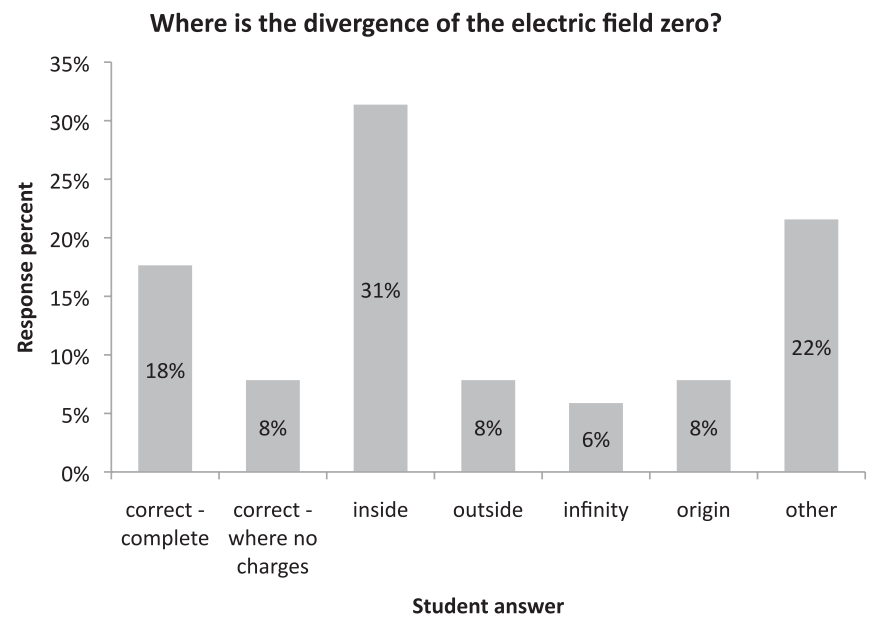

FIG. 4. Student response to a midterm question about a thin shell of uniform charge. $N=51$.
Though we did not perform student interviews related to divergence, students were prompted on their exams with "Explain in words as well as mathematically!" A common explanation for why the divergence was zero inside the sphere, or at the center of the sphere (a total of $38 \%$ of students), was similar to "in the interior $\mathrm{b} / \mathrm{c} \nabla \cdot \mathbf{0}=0$. Outside the shell $\nabla \cdot \mathbf{E} \neq 0$." An analogous argument was made by some students who wrote that the divergence "would vanish at infinity since as $r$ gets really big grad dot $\mathbf{E}$ would get really small and effectively vanish." All of these students were not using appropriate math tools: had they been using either the appropriate Maxwell's equation, or a visual method based on flux, they would not have made these mistakes, and certainly not given the above explanations. By not taking advantage of the visual method to determine divergence, these students are also not accounting for the spatial situation when thinking about the mathematical calculation of divergence. The $14 \%$ of students who chose the origin or infinity, where the value of electric field is zero, may be exhibiting a common introductory-level difficulty of confusing the derivative of a function with its value $[45,46]$.

Unlike the previous section, where students do not make geometry-based symmetry arguments when expected because they likely do not have the relevant tool, students here are not using a tool that they do have: $\nabla \cdot \mathbf{E}=\frac{\rho}{\epsilon_{0}}$. This is a tool that most experts turn to immediately when asked about the divergence of an electrostatic field. It is likely that many students know this equation, but for some reason are not choosing it as a solution method for this problem. Several students with incorrect answers wrote the above equation as part of their work or explanation (4 out of 38 wrong answers and 51 total student answer), making it clear that this is an equation they do know, though they are not using it appropriately. For instance, one student's answer reads

$\nabla \cdot \mathbf{E} \neq 0, \nabla \cdot \mathbf{E}=\frac{\rho}{\epsilon_{0}}, E_{\text {in }}=0$

Div $\mathbf{E}$ inside is zero as $\mathbf{E}=0$

Outside Div $\mathbf{E} \neq 0$ as $\mathbf{E}$ is not zero or uniform $E=\frac{-k q}{r^{2}} \hat{r}$

It seems that the equation relating the divergence of the electric field with $\rho$ is not consistent with this student's idea that divergence is only zero for "zero or uniform" fields. This particular student does not appear to notice the contradiction, and goes with the idea rather than the equation when deciding on an answer. He may be exhibiting a difficulty we have seen consistently when observing students where they think about the common English meaning of the word "divergence" and decide if a field looks diverging rather than applying a rigorous procedure to make a determination. For instance, in response to a clicker question in class about the divergence of a point charge at the origin, one student explained, "I think there is divergence everywhere because the lines are spreading out, but 
I'm not sure what happens at the origin." Students who focus on the English definition of divergence are both failing to access an appropriate mathematical tool and failing to account for the physical geometry when performing a calculation.

In this particular E\&M context $74 \%$ of junior-level students have difficulty applying the concept of divergence. Many of these difficulties seem to be, in part, tied to difficulty accessing all their mathematical tools and trouble accounting for the physical situation when doing a calculation.

\section{Line, surface, and volume integrals}

Many students also have difficulty setting up and correctly computing line, surface, and volume integrals, a difficulty we believe is tied to students failing to visualize the spatial or physical situation when doing an integral. Similar difficulties have been observed for introductory physics students doing 1D integrals [34,47]. As pointed out by Dray and Manogue, in mathematics classes, integrals are viewed very differently than in physics classes $[48,49]$. In the mathematics classes students take in preparation for physics, 1D integrals are nearly always considered to be the area under a curve, and in $3 \mathrm{D}$, surfaces and volumes are always considered in terms of the Cartesian basis vectors and parametrized. On the other hand, in physics, integrals (in any number of dimensions) are considered to be sums of little bits of stuff (mass, charge, $\mathbf{E} \cdot \mathbf{d} \mathbf{A}$, etc.), and the coordinate system for basis vectors is chosen based on the symmetry of the problem. We hypothesize that at the junior level, students are struggling to transition their thinking from the mathematics classrooms' view of integration to the physicists' view.

We have observed while watching students solve problems in homework help sessions and tutorials that students often have difficulty appropriately defining the infinitesimal volume and surface elements, $d V$ and $d A$. Rather than thinking through the specific geometry of the problem, and visualizing a physical small element of volume or surface, students often remember or copy from a text something inappropriate to the particular problem.

For example, when using direct integration to calculate the electric field from a spherical shell of charge, a group of students at a homework help session used $d A=d \phi d \theta$ rather than the correct $d A=r^{2} \sin \theta d \phi d \theta$. It was not immediately obvious to them (by units, for instance) that their expression was wrong, and they were not familiar with how to correct it, or how to test if their $d A$ was correct (i.e., integrate for a whole sphere and see if the result is $A=$ $4 \pi r^{2}$ ). After an instructor explained the correct expression to one student in the group, she had trouble explaining to the rest of the group, which may indicate that this concept is particularly difficult.

Similarly, we find that, when taking a line integral, students often do not visualize a particular path for the line integral, but rather try to solve the problem formulaically. For instance, in one semester students were given a homework question involving the electric field $\mathbf{E}=$ $c(2 x \hat{\mathbf{i}}+z \hat{\mathbf{j}}+y \hat{\mathbf{k}})$ and were asked to "find the potential $V(\mathbf{r})$, using the origin as your reference point (i.e., setting $V(0)=0)$." During homework help sessions, we observed students integrate in one direction only (for instance integrate only in $d x$ ). One student recognized that the integration needed to be done in all directions, but wrote one integral with $d x d y d z$ as a product, instead of the sum of three different integrals, and was then confused because what he had written looked like a volume integral. We observed additional confusion among students about what limits to use in this integration. We observed very few of these struggling students drawing a specific path for this line integral, even though the question included the hint "you must select a specific path to integrate along. It doesn't matter which path you choose, since the answer is path independent, but you can't compute a line integral without having a particular path in mind, so be explicit about that in your solution." Wallace and Chasteen similarly observe that some students do not view the integral in Ampère's law as representing a sum [9]. These student difficulties with line surface and volume integrals likely stem from students failing to visualize the problem and account for the spatial situation when calculating the integral.

\section{POTENTIAL}

The concept and calculation of the scalar potential $V$ underlies a significant portion of an upper-division E\&M course, yet is an issue that students continue to struggle with. Potential is defined as

$$
V(\mathbf{r}) \equiv-\int_{\text {origin }}^{\mathbf{r}} \mathbf{E} \cdot \mathbf{d} l,
$$

but there are many other equations evolving $V$ that are important in an E\&M course at this level:

$$
\begin{gathered}
\nabla^{2} V=\rho / \epsilon_{0}, \\
\mathbf{E}=-\boldsymbol{\nabla} V,
\end{gathered}
$$

and

$$
V(\mathbf{r})=\frac{1}{4 \pi \epsilon_{0}} \int \frac{\rho\left(\mathbf{r}^{\prime}\right)}{\left|\mathbf{r}-\mathbf{r}^{\prime}\right|} d \tau,
$$

for example. One can imagine that this wealth of possibly relevant equations for $V$ may be difficult for students to navigate, and we find that the topic of potential is one in which students have particular difficulty.

\section{A. Evidence that students struggle}

Midterm results.-The same students discussed in Sec. IV B were asked the following on a midterm: 
You have a thin, non-conductive spherical shell with radius $R$ centered at the origin. The shell carries a total charge $-Q$, which is uniformly distributed over the surface of the shell. There are no other charges anywhere. What is the sign of $(V(R)-V(0))$ ?

The correct answer was given by $57 \%$ of these students $(N=51)$. The following student explanation was one of the most clear and correct received:

The electric field is 0 everywhere inside the sphere. Since $V$ is the integral of $\mathbf{E}, V$ will be a constant everywhere inside the sphere. That constant is determined by the boundary condition $V(R)$ therefore: $V(R)=V(0), V(R)-V(0)=0$.

However, $43 \%$ of upper-division students answer this question incorrectly. A breakdown of student responses is shown in Fig. 5. Of students who answered incorrectly, the answers are nearly evenly spit between students who answer "positive" and those who answer "negative." This question was also given during student interviews (during interviews, students were asked the identical question as above but with a shell of positive charge), and on tutorial pre- and posttests, all with similar results.

CUE results. - Students both at CU and at other institutions were asked a similar question related to potential on the CUE diagnostic exam:

You are given a problem involving a non-conducting sphere, centered at the origin. The sphere has a nonuniform, positive and finite volume charge density $\rho(r)$. You notice that another student has set the reference point for $V$ such that $V=0$ at the center of the sphere: $V(r=0)=0$.

What would $V=0$ at $r=0$ imply about the sign of the potential at $r \rightarrow \infty$ ?

(a) $V(r \rightarrow \infty)$ is positive $(+)$

(b) $V(r \rightarrow \infty)$ is negative $(-)$
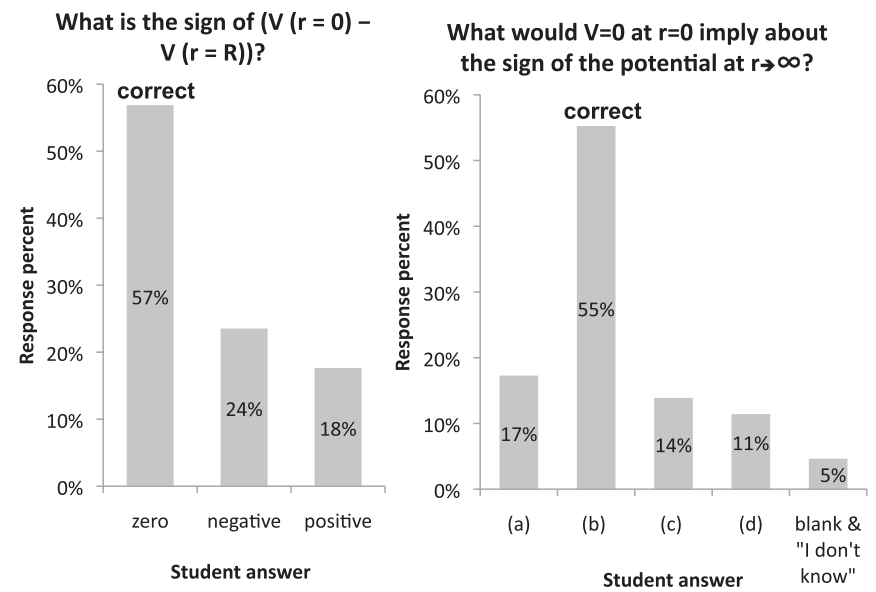

FIG. 5. Left: Student responses to a midterm question about a thin shell of uniform charge. Not included in this graph is one student whose answer was unclear. $N=51$. Right: Student responses to the CUE question about potential described in the text. $N=324$. (c) $V(r \rightarrow \infty)$ is zero

(d) It depends

Briefly explain your reasoning.

Students were graded out of 10 points, 1 of which was for choosing the correct answer, (b). Out of 324 students who answered this question after taking a first semester E\&M course, $55 \%$ chose the correct answer. A breakdown of student responses is shown in Fig. 5. The rest of the points for the above question were given for correctly explaining the physics of positive charges, the definition of potential difference, and connecting these to the answer with reasonable logic. The average overall score on this question was $40 \% \pm 2 \%$.

In student written explanations on the CUE, exams, and tutorial posttests as well as student explanations during interviews, we saw two predominant reasons for incorrect student responses: not accessing appropriate tools for the solution and difficulty setting up or interpreting the problem.

\section{B. Not accessing appropriate tools for the solution}

Several students did not access an appropriate tool to answer the midterm exam question described in the previous section. Rather than choosing an effective tool, e.g., Eq. (1), these students chose one of the many other equations involving $V$. For example, one interview student approached the problem starting with Laplace's equation and then writing down the separation-of-variables solution in spherical coordinates. He then used the usual separationof-variables logic to set the constants for the $1 / r^{\ell+1}$ term equal to zero, and from the remaining equation decided, incorrectly, that this would lead to the difference $V(R)-V(0)=0$ being positive. He later justified this by saying that $V=k q / r$ gives a positive value for a positive point charge. Several students began their solution on the midterm with some version of Eq. (4) (6 out of 22 incorrect answers and 51 total answers), and two others began with the equation for the potential of a point charge, $V=k q / r$.

Several students in written explanations on the tutorial posttest seemed also not to access an appropriate mathematical tool, but rather equated the potential to the charge. For instance, one student wrote "The potential at $R$ is negative because there is a negative charge on the surface. At $r=0$, the potential is zero." Another student wrote on a midterm exam " $V(R)=-Q, V(0)=0$." A student in the CUE validation interviews also simply identified positive charge with positive potential: "as I recall it's a positive relationship, so if the charge is positive $V$ would be positive at infinity" or "since the sphere is positive, $V$ is positive at infinity." This may be similar to the common introductory E\&M student difficulty of associating electric field magnitude at a point directly with the electric potential at that point (rather than with how it is changing) [50]. Another student simply recalled that the potential goes to 
zero at infinity, and applied this even though the given reference point for zero was at the origin.

Other students recalled that there is something arbitrary about potential, but applied this information incorrectly. On the tutorial posttest one student wrote, "It depends on what point you define as zero. If you could define the origin as your zero point or you could define infinity each will give you a different sign," and another explains, "Because $V(r=R)$ is negative, and $V(r=0)$ can be arbitrarily set to zero, the sign of this equation must be negative." In one of the individual interviews, a student explained that the sign of $V(R)-V(0)$ is "a convention thing."

Student written justifications of incorrect answers on the CUE are similar to the student difficulties discussed above. For instance, two students who thought "(c) $V(r \rightarrow \infty)$ is zero" justified their answers with the following explanations:

"The potential is dependent on the $E$ field and as we move towards infinity, the $E$ field drops off and approaches 0 . So this implies the potential does as well."

and

"Voltage at $r=\infty$ is 0 because $\frac{k q q}{r}=0$ as $r \rightarrow \infty$ Integrate from $\infty \rightarrow r$, then $r \rightarrow 0$ to find the potential everywhere."

In the first student explanation, the student may be confusing the value of the function with its derivative, and in the second explanation, the student is starting with the equation for the potential of two point chargescertainly not an appropriate tool to solve this problem.

\section{Difficulty setting up or interpreting the problem}

Other students chose the most effective tool, $V(\mathbf{r}) \equiv$ $-\int_{\text {origin }}^{\mathbf{r}} \mathbf{E} \cdot \mathbf{d} l$, but did not set the equation up correctly (out of 51 total students, 3 out of the 22 incorrect responses included this mistake). For instance, in written explanation on a tutorial posttest, one student wrote "when you integrate the negative $E$ field from infinity to $R$ the sign of the result is positive," when the correct setup gives an integral from 0 to $R$, not infinity to $R$. A couple of students on the exam integrated from infinity to zero. All of these students realized that they need to do a line integral, but incorrectly determined the limits of this integral.

Another interview student accessed the most effective tool, set up the problem correctly, but had difficulty interpreting the result, possibly because she had difficulty connecting the math to the physical situation. An excerpt of her written work during the interviews is shown in Fig. 6. She started by drawing a correct picture of a shell with no electric field lines in the middle, and electric field lines pointing radially outward outside the shell and then wrote a correct equation with the correct limits. She then began to exhibit difficulties. While she had drawn no electric field inside the sphere, and as part of a later explanation

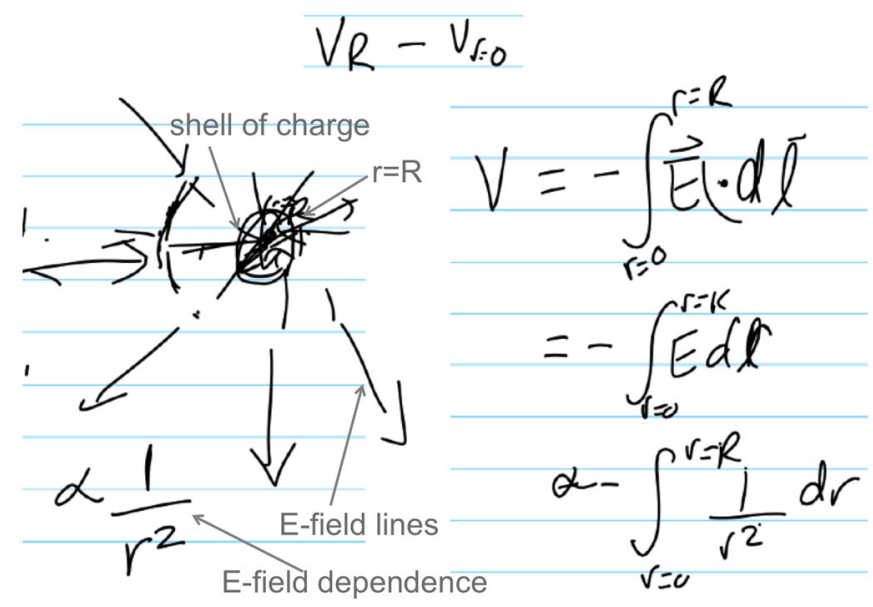

FIG. 6 (color online). Student drawing from interview question about potential. The student's sketch is to the left and her calculations to the right. She originally drew the charged shell as hollow, and explicitly mentioned that $E=0$ there. The scribbles visible in the shell are part of a later explanation.

explicitly said the field inside the shell was zero, she explains (and writes) that $\mathbf{E}$ and $\mathbf{d} l$ are pointing in the same direction, and that $\mathbf{E}$ is proportional to $1 / r^{2}$. She may not be connecting the upper limit of $R$ in her integral to the physical meaning that the integral is entirely inside the shell, or she may not be thinking about the physical situation at all.

Student written work on the midterm exam shows that other students have a similar difficulty. For instance, one student wrote " $(V(r=R)-V(r=0))=-\int_{0}^{R} \vec{E} \cdot \vec{d} l \rightarrow$ opposite the sign of the $E$ field since the surface charge is negative, $V>0$." Again, this student is setting up the problem well, but not interpreting the equation correctly, possibly because he or she is not connecting the equation to the physical situation. Overall, seven students on the midterm exam (32\% of the incorrect responses) set up the correct integral, but failed to interpret it correctly and came to an incorrect conclusion. During CUE validation interviews, some students also exhibited the mistakes listed above (for instance, setting up the line integral correctly, but misinterpreting the results, or not integrating correctly).

Overall, in several different contexts, juniors in E\&M still struggle with the concept and calculation of the electric potential and many of these struggles can be tied to difficulties accessing an appropriate tool out of the many available, and difficulty setting up and interpreting calculations based on physics ideas.

\section{SUMMARY AND DISCUSSION}

We show that some upper-level E\&M students still struggle with fundamental concepts, including Gauss's law, vector calculus, and electric potential. We further show evidence that some of these struggles may be tied 
to student difficulties with mathematics: students have difficulty combining physics ideas with mathematical calculations leading to difficulties setting up and interpreting calculations, students do not account for the underlying spatial situation when performing a calculation, and students do not access an appropriate mathematical tool.

The student difficulties that we observe may hamper student progress on problem solving in multiple areas. Following a framework of the use of mathematics in science developed by Redish[30], we consider that in order to successfully solve a physics problem students must (1) map the physical problem to a mathematical problem, then (2) process and solve the mathematical equations resulting from that mapping, and then (3) interpret the mathematical solution in physical terms. Finally, students must (4) evaluate the final solution to determine if the initial model was accurate. The student difficulties we observe with combining physics ideas with mathematical calculations relate to steps (1) and (3) of Redish's model. The student difficulties accounting for the underlying spatial situation when performing a calculation occur in step (2) of Redish's model, while the difficulty of not accessing an appropriate mathematical tool could occur either in (1) choosing a model or in (2) processing the mathematics. Thus, these difficulties can impede student problem solving at a variety of points. These student difficulties are demonstrated in the three specific contexts of Gauss's law, vector calculus and electric potential.

In the context of Gauss's law we observe the following upper-division difficulties for some students:

- Students make incorrect inferences about the electric field based on Gauss's law. Some students inferred that the electric field at any point on a Gaussian surface is determined only by the charge enclosed, even in nonsymmetric situations.

- Students are unclear in distinguishing flux and electric field.

- Students struggle to articulate complete symmetry arguments. They have particular difficulty with the geometrical symmetry arguments that expert physicists use.

- Students apply Gauss's law when not appropriate.

In the context of vector calculus we observe the following upper-division difficulties for some students:

- Students' understanding of a vector field as a vector with direction and magnitude at every point in space is still somewhat tenuous and students struggle to consistently apply this concept.

- Students struggle with the concept of divergence. They do not consistently connect divergence to a spatial situation, or to the physics of electric fields using a rigorous procedure, but instead may confuse the derivative of a function with its value, or rely on a common English definition of the word divergence.
- Students have difficulty setting up and correctly computing line, surface, and volume integrals; in particular, they do not relate the integral to the physical idea of adding up small pieces.

Regarding the topic of electric potential we observe the following upper-division difficulties for some students:

- Students struggle to choose an appropriate tool for the task out of the many equations related to potential.

- Once they have chosen an appropriate tool, some students struggle to set up or interpret the resulting line integral calculation.

\section{IMPLICATIONS FOR INSTRUCTION}

We have shown that many students have difficulty with mathematics in a junior-level E\&M course, and that these difficulties can be seen in struggles with Gauss's law, vector calculus, and scalar potential. Now that we know some of the difficulties that students have at the junior level, how can we improve instruction to help them succeed?

While we do not know the complete answer to this question, we have evidence that use of our transformed course materials significantly improves student learning in E\&M compared with traditionally taught courses as measured by CUE scores across several semesters of courses both at the University of Colorado and elsewhere [22]. This trend also holds true when many of the specific difficulties brought up in this paper are examined.

For instance, in the CUE question discussed in Sec. III A, $73 \%$ of students in transformed courses recognize a radially symmetric problem as easiest solved using Gauss's law versus only 59\% of students in traditionally taught courses.

Similarly, for the CUE question related to potential discussed in Sec. V, students in traditional courses scored on average $30 \% \pm 3 \%$ and students in transformed courses scored on average $48 \% \pm 3 \%$.

Clearly, using transformed course materials and pedagogy can improve student learning in E\&M. This implies that the use of interactive student-centered materials involving peer-peer interactions, increased opportunities for instructor feedback, and targeted at documented student difficulties is beneficial for upper-division students. Our transformed courses used learning goals developed by a faculty working group, used interactive engagement in classes (in the form of clicker questions with peer instruction and occasional small-group activities), replaced office hours with help rooms centered on students helping each other, added sense-making components to homework, and added an optional weekly tutorial in which small groups worked together on conceptually focused worksheets. For more information on this transformation, see Chasteen et al. [22], and for access to all of our materials, see the CU SEI physics website [51]. 
While transforming our courses to make them more interactive and student centered is clearly a step in the right direction, we still find our students are not completely meeting our goals for them. For instance, $25 \%$ of students in transformed and $24 \%$ of students in traditional classes incorrectly claim that the electric field around the charge distribution in Fig. 1(b) can be computed using Gauss's law. Even though CUE scores on many questions are improved in the transformed courses, student response on many questions is still disappointing. For instance, we are still not satisfied by the score of $48 \% \pm 3 \%$ on the CUE question related to potential. Perhaps even more important than the raw scores is that, when reading through student answers on the CUE, we feel that our students should be able to do better.

This opens the question of why our students are not able to do what we think they should. We consider below both the students and our pedagogy.

Regarding the students themselves, we hypothesize the concepts and mathematical habits of mind discussed in this paper are difficult and take years to develop and that instructors may not remember how difficult these skills are and expect more rapid progress than is reasonable. Also, many of these concepts in E\&M 1 require a combination of challenging skills: using an appropriate mathematical tool, envisioning the spatial situation and connecting it to the mathematics, and translating between physics knowledge and math tools. Each of these is individually difficult, so when combined may become overwhelming even for very good students.

We consider that our pedagogical model may also benefit from some redesign. It is possible that some of the disappointing outcomes are due to a mismatch between the model of instruction and the course at hand. We based our transformations on a successful model from introductory physics classes, and it is possible that, while this model shows improved learning, it is not the most appropriate for upper-division courses populated by physics majors. Perhaps it is more appropriate, at the upper division, to provide interactivity that allows students to engage with more open-ended questions than are afforded by clicker questions and prewritten tutorials. Rather than punctuating lecture with short clicker questions, and long (separate) tutorial activities, lecture could integrate short but focused open-ended group work activities aimed at helping students identify the key skills and ideas on their own. Some examples of this are the paradigms curriculum at Oregon State University (OSU) [52-54] and the OSU Vector Calculus Bridge Project $[49,55]$.

Another potential pedagogical reason that the transformed courses are not as successful as we had hoped for is that, while the transformed course materials are based on observed student difficulties, we had not completed our investigations of student difficulties before creating them. We have learned more about junior-level student difficulties with E\&M material through the process of course transformation. These new insights, including the observations in this paper, have not yet been fed back to further target the course materials at student difficulties, though this is a process that is now beginning.

\section{ACKNOWLEDGMENTS}

This work is funded by CU's Science Education Initiative and by NSF-CCLI Grant No. 0737118. R. E.P. is currently supported by the Miller Institute for Basic Research in Science. We would like to thank Noah Finkelstein for valuable comments on the paper.
[1] L. C. McDermott and E. F. Redish, Resource letter: PER1: Physics education research, Am. J. Phys. 67, 755 (1999).

[2] B.S. Ambrose, Investigating student understanding in intermediate mechanics: Identifying the need for a tutorial approach to instruction, Am. J. Phys. 72, 453 (2004).

[3] T.I. Smith, W. M. Christensen, and J.R. Thompson, Addressing student difficulties with concepts related to entropy, heat engines and the Carnot cycle, AIP Conf. Proc. 1179, 277 (2009).

[4] E. B. Pollock, J.R. Thompson, and D. B. Mountcastle, Student understanding of the physics and mathematics of process variables in $P-V$ diagrams, AIP Conf. Proc. 951, 168 (2007).

[5] D. E. Meltzer, Observations of general learning patterns in an upper-level thermal physics course, AIP Conf. Proc. 1179, 31 (2009).
[6] M. E. Loverude, Student understanding of basic probability concepts in an upper-division thermal physics course, AIP Conf. Proc. 1179, 189 (2009).

[7] T.I. Smith, J.R. Thompson, and D. B. Mountcastle, Addressing student difficulties with statistical mechanics: The Boltzmann factor, AIP Conf. Proc. 1289, 305 (2010).

[8] K. Hayes and M.C. Wittmann, The role of sign in students' modeling of scalar equations, Phys. Teach. 48, 246 (2010).

[9] C. S. Wallace and S. V. Chasteen, Upper-division students' difficulties with Ampère's law, Phys. Rev. ST Phys. Educ. Res. 6, 020115 (2010).

[10] R.E. Pepper, S. V. Chasteen, S. J. Pollock, and K. K. Perkins, Our best juniors still struggle with Gauss's law: Characterizing their difficulties, AIP Conf. Proc. 1289, 245 (2010). 
[11] A. Mason and C. Singh, Do advanced physics students learn from their mistakes without explicit intervention? Am. J. Phys. 78, 760 (2010).

[12] A. E. Kelly, Research as design, Educ. Researcher 32, 3 (2003).

[13] E. Etkina, A. Karelina, S. Murthy, and M. RuibalVillasenor, Using action research to improve learning and formative assessment to conduct research, Phys. Rev. ST Phys. Educ. Res. 5, 010109 (2009).

[14] S. V. Chasteen, K. K. Perkins, P. D. Beale, S. J. Pollock, and C.E. Wieman, A thoughtful approach to instruction: Course transformation for the rest of us, J. Coll. Sci. Teach. 40, 24 (2011).

[15] S. V. Chasteen and S.J. Pollock, Transforming upperdivision electricity and magnetism, AIP Conf. Proc. 1064, 91 (2008).

[16] S. V. Chasteen and S. J. Pollock, A research-based approach to assessing student learning issues in upperdivision electricity \& magnetism, AIP Conf. Proc. 1179, 7 (2009).

[17] S. V. Chasteen and S. J. Pollock, Tapping into juniors' understanding of E\&M: The Colorado Upper-Division Electrostatics (CUE) diagnostic, AIP Conf. Proc. 1179, 109 (2009).

[18] S. Goldhaber, S. Pollock, M. Dubson, P. Beale, and K. Perkins, Transforming upper-division quantum mechanics: Learning goals and assessment, AIP Conf. Proc. 1179, 145 (2009).

[19] M. Dubson, S. Goldhaber, S. Pollock, and K. Perkins, Faculty disagreement about the teaching of quantum mechanics, AIP Conf. Proc. 1179, 137 (2009).

[20] S. J. Pollock, S. V. Chasteen, M. Dubson, and K. K. Perkins, The use of concept tests and peer instruction in upper-division physics, AIP Conf. Proc. 1289, 261 (2010).

[21] S. V. Chasteen, R. E. Pepper, S. J. Pollock, and K. K. Perkins, But does it last? Sustaining a research-based curriculum in upper-division electricity \& magnetism, AIP Conf. Proc. 1413, 139 (2012).

[22] S. V. Chasteen, S. J. Pollock, R.E. Pepper, and K. K. Perkins, "Thinking like a physicist: A multi-semester case study of junior-level electricity and magnetism," Am. J. Phys. (to be published).

[23] A. A. diSessa, Toward an epistemology of physics, Cogn. Instr. 10, 105 (1993).

[24] D. Hammer, Student resources for learning introductory physics, Am. J. Phys. 68, S52 (2000).

[25] D. Hammer, A. Elby, R.E. Scherr, and E.F. Redish, Resources, framing, and transfer, in Transfer of Learning from a Modern Multidisciplinary Perspective, edited by J. P. Mestre (Information Age Publishing, 2005), pp. 89120.

[26] L. C. Hadfield and C. E. Wieman, Student interpretations of equations related to the first law of thermodynamics, J. Chem. Educ. 87, 750 (2010).

[27] T.J. Bing and E.F. Redish, Analyzing problem solving using math in physics: Epistemological framing via warrants, Phys. Rev. ST Phys. Educ. Res. 5, 020108 (2009).

[28] T. Dray and C. A. Manogue, The vector calculus gap: Mathematics $\neq$ physics, Primus 9, 21 (1999).

[29] T. Dray and C. A. Manogue, Bridging the gap between mathematics and physics, APS Forum on Education, http://www.aps.org/units/fed/newsletters/spring2004/ 10oregonstate.html.

[30] E.F. Redish, Problem solving and the use of math in physics courses, arXiv:physics/0608268v1.

[31] J. Tuminaro and E.F. Redish, Understanding students' poor performance on mathematical problem solving in physics, AIP Conf. Proc. 720, 113 (2004).

[32] E.F. Redish, R.E. Scherr, and J. Tuminaro, Reverseengineering the solution of a "simple" physics problem: Why learning physics is harder than it looks, Phys. Teach. 44, 293 (2006).

[33] J. Tuminaro and E. F. Redish, Elements of a cognitive model of physics problem solving: Epistemic games, Phys. Rev. ST Phys. Educ. Res. 3, 020101 (2007).

[34] D. Nguyen and S. N. Rebello, Students' understanding and application of the area under the curve concept in physics problems, Phys. Rev. ST Phys. Educ. Res. 7, 010112 (2011).

[35] D. J. Griffiths, Introduction to Electrodynamics (Prentice Hall, Upper Saddle River, NJ, 1999), 3rd ed.

[36] E. Mazur, Peer Instruction: A User's Manual (Benjamin Cummings, Upper Saddle River, NJ, 1996).

[37] L. C. McDermott and P. S. Shaffer, Tutorials in Introductory Physics (Prentice Hall College Division, Upper Saddle River, NJ, 2001).

[38] These averages are taken by semester. Each semester's course average (over all students in the course that semester) is counted as a single data point to get the listed average. The errors reported are the standard error.

[39] V. K. Otero and D. B. Harlow, Getting started in qualitative physics education research, Rev. PER 2 (2009) [http:// www.compadre.org/per/items/detail.cfm?ID=9122].

[40] C. W. Bowen, Think-aloud methods in chemistry education: Understanding student thinking, J. Chem. Educ. 71, 184 (1994).

[41] C. Singh, Student understanding of symmetry and Gauss's law of electricity, Am. J. Phys. 74, 923 (2006).

[42] C. A. Manogue, K. Browne, T. Dray, and B. Edwards, Why is Ampère's law so hard? A look at middle-division physics, Am. J. Phys. 74, 344 (2006).

[43] S. Rainson, G. Transtromer, and L. Viennot, Students' understanding of superposition of electric fields, Am. J. Phys. 62, 1026 (1994).

[44] A. L. Traxler, K. E. Black, and J. R. Thompson, Students' use of symmetry with Gauss's law, AIP Conf. Proc. 883, 173 (2007).

[45] D. E. Trowbridge and L.C. McDermott, Investigation of student understanding of the concept of velocity in one dimension, Am. J. Phys. 48, 1020 (1980).

[46] D. E. Trowbridge and L.C. McDermott, Investigation of student understanding of the concept of acceleration in one dimension, Am. J. Phys. 49, 242 (1981).

[47] D. Nguyen and S. N. Rebello, Students' difficulties with integration in electricity, Phys. Rev. ST Phys. Educ. Res. 7, 010113 (2011).

[48] T. Dray and C.A. Manogue, Using differentials to bridge the vector calculus gap, Coll. Math. J. 34, 283 (2003).

[49] T. Dray and C. A. Manogue, The vector calculus bridge project, http://www.math.oregonstate.edu/bridge/talks/ OSU.pdf. 
[50] David E. Meltzer, Analysis of shifts in students' reasoning regarding electric field and potential concepts, AIP Conf. Proc. 883, 177 (2007).

[51] University of Colorado, Science Education Initiative, Electricity and Magnetism I: Course Materials, http://www.colorado.edu/sei/departments/ physics_3310.htm.

[52] C. A. Manogue, P. J. Siemens, J. Tate, K. Browne, M. L. Niess, and A.J. Wolfer, Paradigms in physics: A new upper-division curriculum, Am. J. Phys. 69, 978 (2001).

[53] C. A. Manogue and K. S. Krane, Paradigms in physics: Restructuring the upper level, Phys. Today 56, No. 9, 53 (2003).

[54] Paradigms in Physics, http://www.physics.oregonstate .edu/portfolioswiki/.

[55] T. Dray and C. Manogue, Bridging the vector calculus gap, http://www.physics.orst.edu/bridge/. 